DOI: $10.15393 /$ j3.art.2018.5471

The paper is presented at the conference "Complex analysis and its applications" (COMAN 2018), Gelendzhik - Krasnodar, Russia, June 2-9, 2018.

UDC 517.956.223

E. V. TYuRIKOV

\title{
ONE CASE OF EXTENDED BOUNDARY VALUE PROBLEM OF THE MEMBRANE THEORY OF CONVEX SHELLS BY I. N. VEKUA
}

\begin{abstract}
In this paper we obtain results related to the membrane theory of convex shells with piecewise smooth boundary of its median surface. Within this theory we study the problem of realisation of the momentless tense state of equilibrium of the thin elastic shell, the median surface of which is a part of an ovaloid of the strictly positive Gaussian curvature. Development of this theory is based on the usage of generalized analytic functions and is needed for the extended statement of the basic boundary problem. We provide such a further development for a shell with a simply connected median surface using the Riemann-Gilbert special boundary condition. In the paper we identify surface classes for which the index of the corresponding discontinuous boundary condition is efficiently calculated and find sufficent boundary conditions for quasi-correctness of the basic boundary problem in the geometric form.
\end{abstract}

Key words: convex shell, Riemann-Hilbert boundary value problem, index of the boundary value condition

\section{Mathematical Subject Classification: $35 J 56$}

The results considered below originate from two sources: research papers of I. N. Vekua $[8,9]$ into the membrane theory of convex shells with smooth edges (i. e., smooth boundary of their median surfaces) and research papers of A. L. Gol'denveizer [1], who formulated the mathematical statement of the problem of membrane theory for shells with piecewise smooth edges. The method of I. N. Vekua was developed in author's papers $[3,5,7]$ and leads to the statement of the corresponding mathematical

(C) Petrozavodsk State University, 2018 
problem for shells with simply connected median surface. In [7] the author considered two particular cases of the main boundary value problem which are essential for applications. The purpose of this work is the proof of the new sufficient conditions of solvability for the main boundary value problems of the membrane theory of convex shells with piecewise smooth edge formulated in $[1,8]$.

1. Mathematical statement of the problem. In this article we use the terminology and notations introduced in our previous paper [8].

Let $V$ be a thin elastic shell, thereby its median surface is a simply connected surface $S$ with piecewise-smooth edge $L=\bigcup_{j=1}^{n} L_{j}$ and corner points $M_{j}$. We assume that $S$ is the inner part of the surface $S_{0}$ of strictly positive Gaussian curvature of regularity class $W^{3, p}, p>2$, and each of the curves $L_{j}$ belongs to the class $C^{1, \varepsilon}, 0<\varepsilon<1$. Denote by $\boldsymbol{\nu}_{j}^{(k)}(k=1$, 2 ) the vectors tangent to $L$ at the angle point $M_{j}$. The interior angle between these vectors is $\nu_{j} \pi\left(0<\nu_{j}<2\right)$ at this point. The point $M_{i} \in L$ is outbound, if $0<\nu_{j}<1$.

The shell $V$ is a symmetric dome $\left(S^{*}\right.$-dome), if all the corner points $M_{j}$ of the boundary of the median surface are outbound, and the vectors $\boldsymbol{\nu}_{j}^{(k)}$ $(k=1,2 ; j=1, \ldots, n)$ meet at the point $M_{j}$ at equal angles with one of the major directions on the surface.

According to [7], let us consider the problem $T$ of realization of the state of momentless tense equilibrium of a shell under the condition that the projection $\Pi_{\boldsymbol{r}} \boldsymbol{U}(c)$ of the force vector $\boldsymbol{U}(c)$ onto the direction of the vector $\boldsymbol{r}(c)$ is described by the following equation at each point of smoothness of the boundary:

$$
\Pi_{r} \boldsymbol{U}(c)=\sigma(c),
$$

and at each corner from the set $\bigcup_{j=1}^{n} M_{j}$ is described by the equations

$$
\begin{gathered}
\lim _{c \rightarrow M \pm 0} \Pi_{\boldsymbol{r}_{k}} \boldsymbol{U}(c)=\sigma_{k}, \quad(k=1,2), \\
\lim _{c \rightarrow M}|\boldsymbol{U}(c)|=\infty .
\end{gathered}
$$

Here $\sigma(c)$ and $\boldsymbol{r}(c)$ are piecewise continuous scalar function of the point of the contour $L$ and the vector field at the surface $S, \boldsymbol{r}_{k}, \sigma_{k}$ are unilateral limits of the functions $\boldsymbol{r}(c), \sigma(c)$ at the point $M$ in tracing the boundary $L$ in the given direction. 
One can say that equations (2), (3) set the infinite symbolic $V_{\boldsymbol{r}, \sigma}$-vector at the point $M$, introduction of which specifies the condition (3) (the concentration condition, according to the terminology of A. L. Gol'denveizer).

Let us specify now the statement of the problem (1)-(3), adjusting it to the condition of stress concentration (3) using the suitable vector of the vector field $\boldsymbol{r}$.

Let us assume that at each smoothness point the vector $\boldsymbol{r}(c)$ is directed outside the surface $S$, classifying thereby the vector field $\boldsymbol{r}$ on $L$ as admissible. Let $\boldsymbol{r}(c)$ be the admissible vector field $\boldsymbol{r}$ of the singular vector on $L$, setting the continuous field of directions $\boldsymbol{r}(c)$. It is clear that in this case the corresponding vectors $\boldsymbol{r}_{k}(k=1,2)$ are collinear in any point $M$ from the set $\bigcup_{j=1}^{n} M_{j}$.

Let us denote the set of continuous vector fields on $L$ determined by the admissible vector field $\boldsymbol{r}(c)$ by $\mathcal{L}$. The direction of the field $\boldsymbol{l} \in \mathcal{L}$ at the corner point $M$ is called the direction of the generalized normal, if the corresponding vectors $\boldsymbol{r}_{1}, \boldsymbol{r}_{2}$ have different directions.

Definition 1. Let us say that the field of the directions $\boldsymbol{l} \in \mathcal{L}$ belongs to the class $N(M)$, if $\boldsymbol{l}(M)$ is the direction of the generalized normal in the corner point $M$.

Let us assume that the projection $\sigma=\sigma(s)$ of the force vector onto the direction of the Hölder vector $\boldsymbol{r}=\{\alpha(s), \beta(s)\}$ on every curve $L_{j}$ is a function of the positive natural parameter $s$ together with the tangent line and normal component $\alpha(s), \beta(s)\left(\alpha^{2}+\beta^{2}=1 ; \beta \geqslant 0\right)$. Let $J$ be the projection of the surface $S_{0}$ onto the complex plane $z=x+i y$, determined by the selection of the conformal isometric parametrization $(x, y)$ on $S_{0}$; $D=J(S)$ be a field limited by the complex surface $z$ with the boundary $\Gamma=\bigcup_{j=1}^{n} J\left(L_{j}\right)$ and the corner points $\zeta_{j}=J\left(M_{j}\right)$. According to [8], the elliptical system of equations of momentless tense equilibrium of the shell $V$ for the complex function $w(z)$ is the following

$$
w_{\bar{z}}(z)-B(z) \bar{w}(z)=F(z), \quad z \in D
$$

$w_{\bar{z}} \equiv \frac{1}{2}\left(w_{x}+i w_{y}\right), B(z), F(z)$ are function of the class $L_{p}(D), p>2$, on the surface $S$, and the condition (2) is the boundary condition of Riemann- 
Gilbert:

$$
\operatorname{Re}\{\lambda(\zeta) w(\zeta)\}=f(\sigma, K), \quad \zeta \in \Gamma
$$

where

$$
\lambda(\zeta)=\frac{d \zeta}{d s}\left(\beta \frac{d \zeta}{d t}-\alpha \frac{d \zeta}{d s}\right)
$$

$\frac{d \zeta}{d s}=s_{1}+i s_{2}, s_{i}(i=1,2)$ are coordinates of the unit vector tangent to $\Gamma$ at the point $\zeta ; \frac{d \zeta}{d t}=t_{1}+i t_{2}, t_{i}(i=1,2)$ are coordinates of the unit vector on the plane, which is $J$-image of the tangent direction on the surface in the point $J^{-1}(\zeta), K$ is the Gaussian curvature of the surface, superposition $f$ is determined [9] by the statement $T$ and as a function of the Hölder argument $\zeta$ on each curve $L_{j}$. We call the statement (4)-(6) statement $R$, corresponding to the statement $T$.

Let us consider the vector-function $\boldsymbol{s}(\zeta)=\left\{s_{1}(\zeta), s_{2}(\zeta)\right\}, \boldsymbol{t}(\zeta)=$ $=\left\{t_{1}(\zeta), t_{2}(\zeta)\right\}$ on $\Gamma$ denoting left (right) limits of the functions $s(\zeta)$ and $\boldsymbol{t}(\zeta)$ as $s_{j}^{(1)}$ and $t_{j}^{(1)}\left(s_{j}^{(2)}\right.$ and $\left.t_{j}^{(2)}\right)$ accordingly at the point $\zeta_{j}=J\left(M_{j}\right)$ in tracing the field $D$ in the positive direction. Let us set the vector-function $\boldsymbol{\rho}(\zeta)=\left\{\rho_{1}(\zeta), \rho_{2}(\zeta)\right\}$, where $\rho_{1}(\zeta)+i \rho_{2}(\zeta)=\beta \frac{d \zeta}{d t}-\alpha \frac{d \zeta}{d s}$, denoting the right and left limits at the point $\zeta_{j}$ by $\rho_{k}^{(j)}(k=1,2)$. We considered the problem of calculation of the index of boundary condition of the type (5) in [6]. In the same source we provide the proof for the following lemma:

Lemma 1. If the vector field $\boldsymbol{r}(c)$ of the problem $T$ sets the continuous field of directions $\boldsymbol{l}(c) \in \mathcal{L}$, the index to the corresponding problem $R$ in the class of limited solutions is calculated according to the formula

$$
\kappa=-4+\sum_{j=1}^{n}\left[\frac{1}{\pi}\left(\varphi_{j}+\psi_{j}\right)\right],
$$

where $[a]$ is an integer part of $a, \varphi_{j}\left(\psi_{j}\right)$ is the value of the angle between the vectors $\boldsymbol{s}_{j}^{(1)}$ and $\boldsymbol{s}_{j}^{(2)}\left(\boldsymbol{\rho}_{j}^{(1)}\right.$ and $\left.\left.\boldsymbol{\rho}_{j}^{(2)}\right)\right)$, set by the rotation of the first one in the positive direction up to superimposition with the second one.

According to [5], the condition of concentration of stress (3) at the corner point $M_{i}$ when getting to the problem $R$ can be described in the following way

$$
|w(z)| \leqslant K\left|z-\zeta_{j}\right|^{-\alpha_{j}}, \quad 0<\alpha_{j}<1,
$$


where $\alpha_{j}$ is defined by the function $\lambda(\zeta)$. Let us denote the solutions $w(\zeta)$ of the problem $R$, corresponding to the conditions (8), as solutions of the class $H^{*}$ (class of N. I. Muskhelishvili [2]). We need the description of singular nodes (in the sense of N. I. Muskhelishvili) of the coefficient $\lambda(\zeta)$ for the boundary condition (5) for solving the stipulated class of problems.

Suppose $M$ is one of the corner points $M_{i}(i=1, \ldots, n)$ of the boundary of $S^{*}$-dome, $\boldsymbol{\sigma}^{(k)}(k=1,2)$ are the limit values at the point $M$ of the tangent to $L$ of the vector $\boldsymbol{\sigma}(c) ; \boldsymbol{\tau}^{(k)}$ are the corresponding limit values of a singular vector of the normal $\boldsymbol{\tau}(c)$, directed outwards from $S^{*} ; \boldsymbol{k}_{1}, \boldsymbol{k}_{2}$ are the main directions on the surface $S$ at the point $M$; $k_{1}, k_{2}$ are their main curvatures, $k_{1}>k_{2} ; \gamma(\boldsymbol{a}, \boldsymbol{b})$ is the value of the angle between vectors $\boldsymbol{a}$ and $\boldsymbol{b}$ on the surface with the initial point $M$ $(0 \leqslant \gamma(\boldsymbol{a}, \boldsymbol{b}) \leqslant \pi)$. Note that the vectors $(-1) \boldsymbol{\sigma}^{(1)}, \boldsymbol{\sigma}^{(2)}$ create the interior angle at the point $M$, thereby one of the equations is true for that point $\gamma\left(\boldsymbol{\sigma}^{(1)}, \boldsymbol{k}_{m}\right)=\gamma\left(\boldsymbol{\sigma}^{(2)}, \boldsymbol{k}_{m}\right),(m=1,2),\left(0 \leqslant \gamma\left(\boldsymbol{\sigma}^{(k)}, \boldsymbol{k}_{m}\right) \leqslant \pi / 2\right)$. For specification let us consider that

$$
\gamma\left(\boldsymbol{\sigma}^{(1)}, \boldsymbol{k}_{2}\right)=\gamma\left(\boldsymbol{\sigma}^{(2)}, \boldsymbol{k}_{2}\right)=\nu,
$$

$0<\nu \leqslant \operatorname{arctg} \sqrt{\frac{k_{2}}{k_{1}}}, 2 \nu$ is the value of the interior angle in the corner point $M$. Let us denote such a point as $M^{(2 \nu)}$, and the corresponding set (sector) of directions of the generalized normal in this point as $\mathcal{K}^{(2 \nu)}$.

Remark. Direction of the field $\boldsymbol{l}$ in the corner point $M^{(2 \nu)}$ is direction of the generalized normal iff $\gamma\left(\boldsymbol{l}, \boldsymbol{k}_{2}\right) \leqslant \frac{\pi}{2}-\nu$, so that the angle $\gamma\left(\boldsymbol{l}, \boldsymbol{k}_{2}\right)$ between two directions $\boldsymbol{l}$ and $\boldsymbol{k}_{2}$ in the point $M$ is one of the two adjacent angles, the value of which is not bigger than $\frac{\pi}{2}$.

Definition 2. Let us call the direction $\boldsymbol{l} \in \mathcal{K}^{(2 \nu)}$ a singular direction in the point $M^{(2 \nu)}$, if the corresponding point $\zeta=J(M)$ from the set of corner points $\zeta_{j}=J\left(M_{j}\right)(j=1, \ldots, n)$ is a singular node of the problem $R$.

Let us introduce notation for the singular nodes of the problem $R: \boldsymbol{i}, \boldsymbol{j}$ is a pair of ortonormal vectors on the surface $z$, set at the point $J(M)$ by the directions $J\left(\boldsymbol{k}_{1}\right), J\left(\boldsymbol{k}_{2}\right)$ accordingly, thereby the vector $\boldsymbol{i}$ is oriented outside the field $D$; vectors $\boldsymbol{s}^{(1)}, \boldsymbol{s}^{(2)}\left(\boldsymbol{t}^{(1)}, \boldsymbol{t}^{(2)}\right)$ are left and right limits of the vector field $J(\boldsymbol{\sigma}(c)$ ) (field $J(\boldsymbol{\tau}(c))$ ) at the point $\zeta=J(M)$ if we trace 
the curve $\Gamma$ positively. Noting $\nu_{s}=\gamma\left(\boldsymbol{i}, \boldsymbol{s}^{(2)}\right), \nu_{t}=\gamma\left(\boldsymbol{j}, \boldsymbol{t}^{(1)}\right)$, we obtain the equation

$$
\begin{gathered}
\boldsymbol{s}^{(k)}=(-1)^{k} \cos \nu_{s} \cdot \boldsymbol{i}-\sin \nu_{s} \cdot \boldsymbol{j} \quad(k=1,2), \\
\boldsymbol{t}^{(k)}=-\sin \nu_{t} \cdot \boldsymbol{i}+(-1)^{k-1} \cos \nu_{t} \cdot \boldsymbol{j}
\end{gathered}
$$

Now let us consider the point $M^{(2 \nu)}$ and fix some direction $\boldsymbol{l} \in \mathcal{K}^{(2 \nu)}$ of the generalized normal. This direction $S$ in the point $M$ can be set by any of the two collinear vectors $\boldsymbol{r}_{1}(\theta), \boldsymbol{r}_{2}(\theta)$ :

$$
\begin{gathered}
\boldsymbol{r}_{1}=-\sin (\theta+\nu) \boldsymbol{\sigma}^{(1)}+\cos (\theta+\nu) \boldsymbol{\tau}^{(1)}, \\
\boldsymbol{r}_{2}=\sin (\nu-\theta) \boldsymbol{\sigma}^{(2)}+\cos (\nu-\theta) \boldsymbol{\tau}^{(2)},
\end{gathered}
$$

$\theta=\gamma\left(\boldsymbol{r}_{1}, \boldsymbol{k}_{1}\right),-\frac{\pi}{2}+\nu \leqslant \theta \leqslant \frac{\pi}{2}-\nu$. Then the expression for the vectors $\boldsymbol{\rho}_{1}(\theta), \boldsymbol{\rho}_{2}(\theta)$ at the point $M^{(2 \nu)}$, set by the boundary condition (5)-(6), is the following:

$$
\begin{aligned}
& \rho_{1}(\theta, \nu)=\cos (\theta+\nu) \boldsymbol{t}^{(1)}+\sin (\theta+\nu) \boldsymbol{s}^{(1)}, \\
& \rho_{2}(\theta, \nu)=\cos (\nu-\theta) \boldsymbol{t}^{(2)}-\sin (\nu-\theta) \boldsymbol{s}^{(2)},
\end{aligned}
$$

thereby $\boldsymbol{s}^{(1)} \cdot \boldsymbol{s}^{(2)}<0, \boldsymbol{t}^{(1)} \cdot \boldsymbol{t}^{(2)}<0, \boldsymbol{s}^{(1)} \cdot \boldsymbol{t}^{(2)}<0, \boldsymbol{s}^{(1)} \cdot \boldsymbol{t}^{(2)}=-\boldsymbol{s}^{(2)} \cdot \boldsymbol{t}^{(1)}$. Lemma 2. There is the unique value $\mu \in\left(0, \operatorname{arctg} \sqrt{\frac{k_{2}}{k_{1}}}\right)$ with such properties that the direction $\boldsymbol{k}_{1}$ of the generalized normal in the point $M$ is a special direction of the point $M^{(2 \mu)}$.

Proof. In our case, according to the definition of the singular node [2] of the problem $R, \varphi+\psi=\pi, \varphi$ and $\psi$ are defined by Lemma 1 . If the vector $\boldsymbol{r}(\theta)$ sets the direction $\boldsymbol{l}$ of class $\mathcal{K}^{(2 \nu)}$, then the collinearity of the vectors $\boldsymbol{\rho}_{2}(0, \nu)$ and $\boldsymbol{s}_{1}$ follows from the latter equation. As the vector $\boldsymbol{r}(0)$ corresponds to the collinear direction $\boldsymbol{k}_{1}$ and $\left|\boldsymbol{\rho}_{1}(0, \nu)\right|=\left|\boldsymbol{\rho}_{2}(0, \nu)\right|$, then, taking into account (9), (10), we get

$$
\sin \nu \cdot \sin 2 \nu_{s}=\cos \nu \cdot \cos \left(\nu_{s}+\nu_{t}\right) .
$$

As according to the well-known property of conjugate isometric parametrizations [9, Chapter 2],

$$
\sin \nu_{t}=\left(1+\frac{1}{\alpha} \operatorname{ctg}^{2} \nu\right)^{-\frac{1}{2}}, \sin \nu_{s}=\left(1+\alpha \operatorname{ctg}^{2} \nu\right)^{-\frac{1}{2}} \quad\left(\alpha=\frac{k_{2}}{k_{1}}<1\right)
$$


then, from (11), after a number of transformations with the subsequent substitution $\operatorname{ctg}^{2} \nu=t$, we get the equation

$$
\frac{1}{2 \sqrt{\alpha}} \cdot(t-1)=\left(1+\alpha^{-1} t\right)^{\frac{1}{2}}(1+\alpha t)^{-\frac{1}{2}} .
$$

Basing on the obvious graphic considerations, we make the conclusion that the equation (12) has the singular route $t=t_{0}<\alpha^{-1}$; this implies the statement of the lemma.

Lemma 3. There is the unique value $\eta \in\left(\operatorname{arctg} \sqrt{\frac{k_{2}}{k_{1}}}, \frac{\pi}{2}\right)$ such that the direction $\boldsymbol{k}_{1}$ of the generalized normal at the point $M$ is a singular direction of the point $M^{(2 \eta)}$.

Proof. If $\eta>\operatorname{arctg} \sqrt{\frac{k_{2}}{k_{1}}}$, then the direction of the vector $\boldsymbol{k}_{1}$ in the point $M^{(2 \eta)}$ is singular under the condition $\varphi+\psi=0$, which is possible only in the case of orthogonality of the vectors $\boldsymbol{\rho}_{2}(0, \nu)$ and $\boldsymbol{s}^{(1)}$. Hence, taking into account the equations (12), we deduce the equation

$$
\sin \nu \cdot \cos 2 \nu_{s}=-\cos \nu \cdot \sin \left(\nu_{s}+\nu_{t}\right) .
$$

After the corresponding transformations with the subsequent substitution $t=\operatorname{tg}^{2} \nu$, we get the equation $(\alpha+1)^{-1}(t-\alpha)=(\alpha t+1)^{-\frac{1}{2}}(t+\alpha)^{\frac{1}{2}}$ with the singular route $t=t_{0}>1$.

Let us consider the symmetric point $M^{(2 \nu)}$ from the number of the corner points $M_{i}(i=1, \ldots, n)$ and the function

$$
\chi(\theta, \nu) \equiv \varphi(\nu)+\psi(\theta, \nu) \quad\left(|\theta|<\frac{\pi}{2}-\nu, \quad 0<\nu \leqslant \operatorname{arctg} \sqrt{\frac{k_{2}}{k_{1}}}\right),
$$

corresponding to it, in which $\varphi \equiv \varphi(\nu), \psi \equiv \psi(\theta, \nu)$ accordingly coincide with the values $\varphi_{i}, \psi_{i}$, set at the point $M_{i}$ by the equation (7). Let us note that the function $\psi(\theta, \nu)$ is even, according to the argument $\theta$. The following statements directly follow from Lemma 2

Statement 1. $\pi<\chi(0, \nu)<2 \pi$, if $0<\nu<\mu$, and $0<\chi(0, \nu)<\pi$, if $\mu<\nu<\eta$; here $\mu, \eta$ are the numbers defined in lemmas 2 and 3, $0<\chi(0, \nu)<\pi$, if $\eta<\nu<\frac{\pi}{2}$. The following statement also holds true. 
Statement 2. The function $\psi(\theta, \nu)$ for any $\nu \in\left(0, \operatorname{arctg} \sqrt{\frac{k_{2}}{k_{1}}}\right)$ monotonously decreases on $[0, \nu]$ for any argument $\theta$; thereby, $\psi\left(\frac{\pi}{2}-\nu, \nu\right)>\pi$ $\forall \nu \in(0, \mu)$, where $\mu$ is defined by Lemma 2. The proof of Statements 1 and 2 is made according to the scheme [4] with inconsiderable changes.

Remark. Monotonous decreasing of the function $\psi(\theta, \nu)$ is set in the similar way for any $\nu \in\left(\operatorname{arctg} \sqrt{\frac{k_{2}}{k_{1}}}, \frac{\pi}{2}\right)$ as the function of the argument $\theta$.

Let us consider a $S^{*}$-dome with the corner points $M_{i}(i=1, \ldots, n)$ of the boundary and introduce the following notation: $\boldsymbol{k}_{1}^{(i)}, \boldsymbol{k}_{2}^{(i)}$ are the major directions on the surface $S_{0}$ at the point $M_{i}, k_{1}^{(i)}, k_{2}^{(i)}$ are the major curvatures corresponding to them $\left(k_{2}^{(i)}<k_{1}^{(i)}\right)$. Let us denote the numbers defined in the points $M_{i}(i=1, \ldots, n)$ by lemmas 2 and 3 , accordingly, by $\mu_{i}, \eta_{i}$. Let us refer the projecting part $M_{j}$ to 1 -type (2-type, 3 -type), if the condition $0<\pi \nu_{j}<2 \mu_{j}\left(2 \mu_{j}<\pi \nu_{j}<2 \eta_{j}, 2 \eta_{j}<\pi \nu_{j}<\pi\right)$ holds true for the value $\nu_{j} \pi$ of the interior angle. Let us consider the vector field $\boldsymbol{l}$, belonging to the class $N\left(M_{i}\right)$ in every point $M_{i}(i=1, \ldots, n)$. Let us denote the class of such fields by $N\left(S^{*}\right)$.

Lemma 4. If $n^{(k)}$ is the number of corner points of the $k$-type $(k=1,2$, $3 ; n^{(1)}+n^{(2)}+n^{(3)}=n$ ) of the boundary $L$, then the index of the boundary condition of the problem $R$ in the class $H^{*}$ is calculated according to the formula

$$
\kappa=2 n^{(1)}+n^{(2)}-4
$$

$\forall \boldsymbol{l} \in N\left(S^{*}\right)$.

The statement of the lemma follows from (7) and Statements 1-2.

\section{Formulation of the outcomes.}

Definition 3. Let us call the problem $T$ unconditionally solvable for the given field of the directions $\boldsymbol{l} \in \mathcal{L}$ and $B_{l}^{*}$-solvable, if the problem $R(T)$ is unconditionally solvable in the class $H^{*}$ the same as the problem of Riemann-Gilbert with inhomogeneous boundary condition. Let us also say that the equilibrium condition $B_{l}^{*}(m)$ is observed for the dome $S^{*}$, if the solution of the problem $R(T)$ depends on $m$ random real-valued parameters. 
Theorem 1. If $s=2 n^{(1)}+n^{(2)} \geqslant 3$, then $B_{l}^{*}(s)$-state is observed for any $\boldsymbol{l} \in N\left(S^{*}\right)$ of $S^{*}$-dome.

Theorem 2. If $\pi \nu_{i_{k}}$ coincides with one of the numbers $2 \mu_{i_{k}}, 2 \eta_{i_{k}}$ in the point $M_{i_{k}}(k=1, \ldots, m)$, then $B_{l}^{*}(s)$-state is observed for any $\boldsymbol{l} \in N\left(S^{*}\right) \backslash \bigcup_{k=1}^{m}\left\{\boldsymbol{k}_{1}^{\left(i_{k}\right)}\right\}$.

The validity of Theorems 1, 2 follows from Lemmas 2, 3 and outcomes [3].

The following theorem ensues from Theorem 1:

Theorem 3. If among the corner points of the boudary $L$ the points of 1-type are missing, and the number of corner points of 2-type does not exceed two, then the problem $R$ is well-defined.

In the conclusion let us note that $\mu_{i}=\frac{\pi}{6}, \eta_{i}=\frac{\pi}{3}$ in an ombilic point $M_{i}$, and any direction of $\boldsymbol{l}$ class $N\left(M_{i}\right)$ is singular.

\section{References}

[1] Gol'denveizer A.L. On the application of the Riemann-Hilbert problem solutions to the calculation of membrane shells. Prikl.Mat.Mekh. 1951, vol. 15 , no. 2, pp. $149-166$.

[2] Muskhelishvili N. I. Singular integral equations. Fizmatgiz, Moscow, 1968. pp. 511. (in Russian).

[3] Tyurikov E. V. Boundary problems of the theory of infinitesimal bending of the surfaces of positive curvature with piecewise smooth edge. Sbornik: Mathematics, 1977. vol. 103(145), no. 3 (7), pp. 445-462.

[4] Tyurikov E. V. Boundary problem of membrane theory of convex shells for one class of symmetric domes. Izv. vuzov. North Caucasus region. Natural sciences, 2018, no. 1, pp. 49-54. DOI: https://doi.org/10.23683/ 0321-3005-2018-1-49-54.

[5] Tyurikov E. V. Geometric Analogue of the Vekua-Goldenveizer Problem. Doklady Russian Academy of Sciencess, 2009. vol. 424, no 4, pp. 445 457.

[6] Tyurikov E. V. On one boundary problem of the theory of infinitesimal bends of the surfaces. Vladikavkaz Mathematical Journal, 2007. vol. 9, no. 1, pp. $62-68$.

[7] Tyurikov E. V. About one special riemann-hilbert problem and its application. Izv. vuzov. North Caucasus region. Natural sciences., 2016. no. 4, pp. 31-35. DOI: https://doi.org/10.18522/0321-3005-2016-4-31-35. 
[8] Vekua I. N. Generalized analytic functions. Fizmatgiz, Moscow, 1959. (in Russian).

[9] Vekua I. N. Some General Methods for Constructing Versions of Shell Theory. Fizmatgiz, Moscow, 1982. (in Russian).

Received May 29, 2018.

In revised form, September 7, 2018.

Accepted September 10, 2018.

Published online September 25, 2018.

Don State technical University

1 Gagarin pl., Rostov on Don 344000, Russia

E-mail: etyurikov@hotmail.com 